\title{
LOS TEXTOS ESCOLARES PARA PRIMER AÑO EN EL CICLO BÁSICO UNIFICADO. ESTUDIO DE CASOS EN RÍO NEGRO (1989-1995)
}

\author{
Nancy Liliana Salerno (UNCOMAHUE) ${ }^{*}$
}

\begin{abstract}
Resumen
Este artículo es una aproximación al estudio de los textos escolares correspondientes al $1^{\circ}$ año del Ciclo Básico Unificado - CBU-, en escuelas de nivel medio de la ciudad de Cipolletti, en el período 1989 a 1995. Estas producciones suplieron la ausencia de libros o manuales, ya que en las esferas editoriales prevalecían otros lineamientos que no se ajustaban a los encuadrados en la reforma educativa de Río Negro. En esta instancia se enfatiza en las características de los textos referidos a Historia Local y Regional, su adecuación pedagógica, los modos de elaboración, su difusión entre los alumnos y los docentes, como también, el enfoque epistemológico empleado fundamentalmente en el área llamada "Mundo Socio-cultural" que estaba constituida por tres asignaturas: Historia, Geografía y Educación Cívica.
\end{abstract}

\section{Palabras Clave}

Reforma educativa - Ciclo básico unificado -Textos escolares.

\begin{abstract}
This article is an approximation to the study of the school texts corresponding to the $1^{\circ}$ year of the Basic Unified Cycle - CBU-, in schools of average level of Cipolletti's city, in the period 1989 1995. These productions replaced the absence of books or manuals, since in the publishing spheres there were prevailing other limits that were not adjusting to the fitted ones in the educational reform of Rio Negro. In this instance it is emphasized in the characteristics of the texts adapted of Local and Regional History, their pedagogical adaptation, modes of production, its dissemination between students and teachers, as well as the epistemological approach used primarily in the
\end{abstract}

Especialista en Historia Económica y de las Políticas Económicas. Facultad de Economía y Administración de la Universidad Nacional del Comahue y Universidad Nacional de Buenos Aires. Profesora en Historia. Facultad de Humanidades. Universidad Nacional del Comahue. 
area called " Sociocultural World " which was incorporated three subjects: History, Geography and Civic Education.

\section{Key words}

Educational reform - Basic unified cycle - School texts.

\section{Introducción}

El presente trabajo forma parte del proyecto "El libro de texto como constructor de representaciones: la imagen de la Patagonia. Río Negro y Neuquén (1960-2000)", dirigido por la Dra Mirta Teobaldo y la Mag. Amelia García, pertenecientes a la Facultad de Ciencias de la Educación (UNComahue), Cipolletti.

Pretende abordar el estudio de la producción de textos escolares, en el marco de la Reforma Educativa del Nivel Medio en Río Negro, que se implementó en algunos establecimientos de Cipolletti, específicamente CBU № 15 , $N^{\circ} 5$ y N ${ }^{\circ} 17$ en el período 1989-1995. Se refiere al trabajo de docentes del Ciclo Básico Unificado, centrado en el primer año, dado que el cambio curricular implicaba el análisis de la realidad local y regional (Alto Valle-Patagonia), con un enfoque interdisciplinario. Estas producciones resultaron del trabajo en equipo de docentes con una concientización y compromiso en la tarea emprendida, lo que aseguró la retroalimentación constante de la práctica escolar -praxis-, con la planificación y evaluación del grupo en instancias o espacios creados con este fin. Asimismo, estas producciones suplieron la ausencia de libros o manuales de textos, ya que en las esferas editoriales prevalecían otros lineamientos que no se ajustaban a los encuadrados en la reforma educativa de Río Negro.

En esta instancia se realizará una primera aproximación a la problemática planteada, con el objetivo de estudiar las características de los textos y su adecuación pedagógica, los modos de elaboración, su difusión entre los alumnos y los docentes, como también, el enfoque epistemológico empleado fundamentalmente en el área llamada "Mundo Socio-cultural" que estaba constituida por tres asignaturas: Historia, Geografía y Educación Cívica. En este caso particular, se tomarán textos seleccionados y/o especialmente adaptados de Historia Local y Regional para el primer año que fueron utilizados en distintos CBU de la ciudad de Cipolletti, por grupos de docentes que variaban de año en año y modificaban enriqueciendo el material didáctico (Cuadernillo de Historia Regional, 1990). La experiencia comenzó en el CBU No 15 durante 1989, y en los años siguientes fueron enriquecidos y empleados en otros establecimientos, con equipos formados por distintos profesores del Área Mundo Socio-Cultural.

En lo que se refiere a las fuentes utilizadas, a la escasa bibliografía general y específica sobre estas temáticas locales y regionales, se suman publicaciones 
de FLACSO, informes docentes, de textos seleccionados, trabajos prácticos, planificaciones anuales y de clases, diversas ponencias, y trabajos de tesis relacionadas a la reforma educativa.

\section{Implementación del Ciclo Básico Unificado como parte de la Reforma Educativa de Río Negro}

Según el equipo de investigación dirigido por Carlos Oyola (1988) sobre la Reforma del C.B.U, se había implementado durante 1986 en forma experimental un nuevo diseño curricular en una escuela de la localidad de El Bolsón y en otra del Barrio Melipal de San Carlos de Bariloche, bajo el gobierno provincial de Álvarez Guerrero en consonancia con el regreso de la democracia a través de Raúl Alfonsín. Paralelamente, se desarrollaría un cronograma tentativo con cinco fases en Río Negro para concretar el "Congreso Pedagógico Nacional" (Revista "Puerta Abierta", 1986).

La Resolución № 964/86 constituyó el soporte jurídico- normativo del CBU y destaca que luego de 1987 se extendió a establecimientos del nivel en Choele Choel, Viedma, Bariloche, San Antonio y Villa Regina, hecho que comienza a concretarse en la gestión Massaccesi, para finalmente tomar un carácter obligatorio a partir de 1988.

De este modo, en algunos establecimientos educativos de nivel medio de Cipolletti se comenzó con la implementación del CBU al iniciar el año 1988 y se aplicó sólo en los primeros años para ir gradualmente avanzando. Es así como, a partir de 1991 se logró implementar el Ciclo Superior Modificado para cuarto y luego quinto año.

Es importante considerar, que entre los años 1989-1999, la presidencia de nuestro país fue ocupada en dos períodos consecutivos por Carlos Saúl Menem, a partir de una reforma constitucional en el año 1994 que permitió su reelección. En los años noventa se consolidaron las tendencias neoliberales prefijadas desde mediados de los setenta. Las diversas políticas adquirieron la forma de un conjunto orgánico, sistemático y funcional al programa de reestructuración del Estado y la economía llevado adelante (Rapoport, 2003). Es en este escenario, que la Reforma del Nivel Medio en Río Negro bajo el gobierno de Pablo Verani encuentra su fin; y regresó a partir de 1996, el sistema tradicional caracterizado por una estructura atomizada, prevaleciendo el individualismo sobre el trabajo en equipo de los docentes. Este proceso no implicó un cambio de los contenidos curriculares que se mantuvieron poniendo el énfasis en el medio local y regional para primer año, el medio nacional para segundo año, el espacio latinoamericano para tercer año, los distintos modos de producción en Europa Occidental en cuarto y el análisis de problemáticas nacionales y latinoamericanas para quinto. Esto está en proceso de transformación con la nueva Reforma que ya está en vigencia. 


\section{Relación trabajo docente, textos y las formas de utilizarlos}

El área Mundo Socio Cultural, como se afirmó anteriormente, estaba formada por tres asignaturas: Historia, Geografía y Educación Cívica; en conjunto apuntaban a la formación de una conciencia nacional dentro de un marco latinoamericano, para que el adolescente tenga una visión global e interprete los hechos de la realidad social en forma crítica y reflexiva; tenga actitudes democráticas; valore a la persona como tal; adquiera los instrumentos necesarios para desenvolverse en forma independiente en el abordaje de nuevas temáticas; y se comprometa en la transformación de la realidad.

El eje conceptual interdisciplinario era el punto de partida como centro integrador, y en el caso específico del Área Mundo Socio-Cultural para primer año era "la relación del hombre con el medio local y regional" y se perseguía como objetivo general, comprender y valorar la organización del conjunto de condiciones naturales y culturales locales y regionales (Luc, 1989). Los objetivos promocionales eran conocer e interpretar el medio en que se vive y aplicar los conocimientos adquiridos a situaciones nuevas, según se desprende del Documento No 9 (1988) "La Fundamentación del Área Mundo Socio Cultural. Diseño Curricular del Nivel Medio", Consejo Provincial de Educación de Río Negro. No se delinearon puntualmente los ejes conceptuales didácticos porque se pretendía un curriculum descentralizado atendiendo a la democratización de la enseñanza y a las necesidades de cada zona.

Para concretar esta reforma, se crearon cargos que incluían talleres para docentes. El taller de educadores consistió en un espacio de dos horas cátedras de duración que reunía una vez por semana a todos los profesores abocados en un primer momento al primer año de la experiencia. Allí, se analizaba la práctica docente, lo que permitía la comunicación abierta y el descubrir las miradas o los enfoques que llevaban a seleccionar los contenidos a dar, como una serie de estrategias o abordajes en las distintas áreas. Según las entrevistas realizadas, se designaba a un docente coordinador y a otro que llevaba el registro para posteriormente analizar en otras reuniones, con coordinadores de otros C.B.U. Raúl Ageno lo caracteriza como el "espacio" en el que, mediante la investigación conjunta, los docentes analizan su propia práctica resignificándola (Achilli, Ageno y Ossana, 1988).

En estas instancias de análisis de la práctica educativa se trabajaron y pautaron los criterios de selección y organización de contenidos para evitar la liberalización del proyecto y desvirtuarlo. Los contenidos debían responder a la necesidad, realidad e intereses de cada grupo y que docentes y alumnos elaboraran los contenidos convirtiéndose en protagonistas del proceso de aprendizaje. Los criterios para tal selección y jerarquización eran: afianzar la identidad nacional donde hechos, conceptos, sistemas y procedimientos sean vistos desde lo nacional y latinoamericano; reafirmar y profundizar actitudes democráticas; atender a los intereses, necesidades y realidades propias del 
adolescente; y proponer contenidos integradores y dialécticos (Lanza y Finocchio, 1993).

Este proceso fue acompañado por una Comisión de Perfeccionamiento Docente C.B.U., y la Facultad de Ciencias de la Educación de la Universidad Nacional del Comahue (UNCo). El ofrecimiento a los docentes de Nivel Medio resultó variado: un programa de perfeccionamiento con jornadas de varios meses de duración y años consecutivos; seminarios en 1991 inscriptos en el Programa de Mejoramiento de la Calidad de la Educación en los CBU de Río Negro en el marco del convenio FLACSO- CPE; Jornadas-Talleres de articulación entre primaria y secundaria desde el gremio docente, Unión de Trabajadores de la Educación de Río Negro (Un.T.E.R.), en 1990 y aprobados por la Dirección de Formación, Capacitación, Perfeccionamiento y Actualización Docente (DiFoCaPeA); seminarios en distintos departamentos de la Facultad de Humanidades de la UNCo desde 1991. En este mismo marco, es necesario enfatizar la importancia del seminario de Historia Regional que comenzaba a dictarse por aquellos años. Todos estos espacios no tuvieron un carácter obligatorio para los docentes a pesar de las reuniones periódicas entre los coordinadores de los distintos Talleres de Educadores y el Servicio de Apoyo Técnico.

Paralelamente funcionaba el Taller de Área como espacio institucional de dos horas cátedras que permitía en este caso, a los profesores de Geografía, Historia y Educación Cívica reunirse y acordar criterios, en base a recortes de la realidad local y regional, para seleccionar los contenidos significativos desde las tres asignaturas con la autonomía necesaria y la capacidad de cada uno para que los enfoques resultaran interdisciplinarios y de ninguna manera una disciplina se subordinara a otra. Era responsabilidad de estos equipos planificar en estos espacios, los encuentros de los Talleres Integrales que tenían una duración cuatrimestral, eran optativos para los alumnos, y trataban temáticas que surgían de problematizaciones comunes a la sociedad siendo pertinentes su abordaje desde el área.

En este taller, tres preguntas resultaron claves: qué, para qué y cómo se enseña. El año lectivo se hallaba dividido en dos cuatrimestres, por lo tanto, las experiencias de trabajo grupal determinaron que en cada establecimiento - CBU se planificara de una determinada manera. En el análisis de algunas planificaciones se observa que hubieron docentes que priorizaron el espacio patagónico, luego el Alto Valle, y posteriormente trataron problemáticas locales. Otros equipos partieron desde el análisis del Alto Valle, incluían su medio local, y a continuación abordaron la región patagónica. Mientras otros partieron del medio local, luego el Alto Valle y finalmente la Patagonia.

La propuesta de otro modelo generó situaciones de disenso, ante la complejidad, la pluralidad de enfoques y, fundamentalmente, ante la ausencia de libros o manuales escolares editados acordes con los nuevos lineamientos curriculares propuestos por la Reforma (Rodríguez y Dobaño Fernández, 2001). Esto sucedió 
en un contexto donde el Estado dejó de regular la producción de libros de textos a través de las Reglamentaciones, y de dar su aprobación mediante el trabajo de las Comisiones evaluadoras a partir de 1983 en el ámbito de la Capital Federal y poco después en las jurisdicciones provinciales (Linares, 2009).

Los libros o manuales de texto no respondían a los avances y las nuevas contribuciones desde las Ciencias Sociales, que implica el abordaje de lo regional, enmarcado en contextos mayores, más específicamente el plano nacional e internacional. Asimismo, la enseñanza en torno a recortes de la realidad con problemáticas cercanas en el tiempo y en el espacio, conducía a los docentes a periodizar para un mejor análisis de los elementos contextuales.

Todo texto escolar debe articular intencionalidades educativas con determinados contenidos disciplinares y con una teoría pedagógica explícita o implícita. En este caso, es necesario señalar que fue producción propia según el constructivismo como teoría del aprendizaje, el que se afianzaba en el cuerpo docente (Allal, 1979; Casávola y Castorina, 1980; Novak, 1980; Novak y Gowin,1981; Moreira,1988). En consecuencia, los contenidos más significativos, válidos socialmente, debieron ser seleccionados y organizados en base a ejes centrales o estructurantes producidos en relación a problemáticas de la realidad social, y de este modo, formaron parte del proceso educativo ingresando por primera vez en la escuela media.

La tarea no resultó fácil de resolver para los docentes, ni para los editores que deben renovar los textos escolares. Los Libros del Quirquincho constituyeron una respuesta a algunas de las necesidades de aquellos años; en especial la colección "La otra historia" de Miguel Ángel Palermo o "La Patagonia" de Hebe Clementi (1991) escrito para chicos. Merece destacarse el aporte realizado por especialistas del Área "Educación y Sociedad" de la Facultad Latinoamericana de Ciencias Sociales, (FLACSO) que elaboraron una serie de cuadernillos para utilizar en el trabajo y se distribuyeron en las distintas regionales, como se explicará posteriormente.

Ante la responsabilidad social de los docentes para integrar a los alumnos en la comunidad del conocimiento, se idearon distintas estrategias:

a.- Elaboración de prácticos en forma interdisciplinaria con una selección de pasajes procedentes de autores pertinentes al tema tratado.

b.- Elaboración del material de estudio por asignatura sin forzar los tiempos parciales, pero con un abordaje en conjunto al cierre del cuatrimestre.

El presente trabajo, como se mencionó anteriormente, responderá a esta última modalidad: cuadernillos de Historia Regional con textos seleccionados y/o especialmente adaptados para el primer año que fueron utilizados en distintos CBU de la ciudad de Cipolletti. Al analizar estos textos, siguiendo el trabajo de Rinaudo y Galvalisi (2002) se desprenden las siguientes características:

- Predominaban los textos históricos aunque se incluyeron algunos de las otras dos disciplinas, como se podrán leer a posteriori. 
- Estos textos que comenzaban siendo cortos eran adaptados para una edad evolutiva de 12 o 13 años, continuamente perfeccionados o enriquecidos con el aporte de otros docentes ya que respondía a un sistema abierto y en continua variación, en los sucesivos años. En consecuencia, los contenidos estaban actualizados, representando los últimos enfoques o corrientes promovidas desde los medios académicos, como se tratará posteriormente.

- Algunos textos eran de autoría propia, síntesis de lecturas previas de fuentes primarias y secundarias; otros fueron adaptaciones resumidas de textos, mientras que también se adjuntaron textos de origen. En el cuadernillo se consignaba la bibliografía final sin especificar las fuentes empleadas para cada texto.

- Se utilizó bibliografía de distinto tipo en los primeros años, tratando que fuera lo más actualizada posible. Había que redactar haciendo adaptaciones en los aprendizajes escolares adecuando el nivel académico al nivel escolar para evitar posibles fracasos en el aprendizaje de los alumnos.

- Utilizaban expresiones simples y claras con un buen tamaño de la letra.

- Se atendió el problema de la legibilidad de estos textos escolares que resultaron necesarios en la alternativa para aprender intentando un camino para reafirmar el papel social del saber y su compromiso con la vida.

- No se utilizaron ilustraciones: dibujos, gráficas, y figuras por razones económicas. A través de las entrevistas/carpeta de alumnos, se confirma la utilización de copias mimiográficas para abaratar costos y en años posteriores fotocopias. Una de las primeras modalidades fue la entrega individual de cada texto para proceder a la lectura comprensiva en las clases.

El aprendizaje era una preocupación constante en quienes redactaban los textos, se incluían cuestiones interesantes para reflexionar y una lógica muy clara de la secuencia de presentación de cada uno de los temas. Al considerar entrevistas orales a actores esenciales del proceso de CBU -1989 a 1995 se comprobó que cada profesor tenía la libertad de trabajar el texto a su criterio. A pesar de esto y de las nuevas tendencias didácticas, sin embargo, se apelaba a viejos recursos que son continuidades que indican la pervivencia de métodos exitosos, como la lectura modelo propia del normalismo, para luego proceder a la lectura en voz alta por parte de los alumnos, la confección del vocabulario básico con el uso de diccionarios, la identificación de palabras claves, el subrayado de las ideas formando oraciones, anotaciones marginales o en interlíneas, titulados de párrafos, resumen, cuadros sinópticos, esquemas de contenidos e incluso el empleo y la elaboración de mapas conceptuales, a partir del año 1991. Se complementaba con otras estrategias y recursos didácticos, como se explicará posteriormente. 


\section{Estudio de casos o experiencias}

En los distintos años de la experiencia, se fueron perfeccionando los ejes interdisciplinares, de acuerdo al análisis de sucesivas planificaciones del área y entrevistas realizadas. En 1989 se acordó en el CBU № 15 partir de un interrogante para el primer cuatrimestre: ¿crecimiento o estancamiento del Alto Valle como oasis agrícola?; y para el segundo fue ¿aislamiento de la región patagónica?. En los años posteriores y en otros CBU ( $N^{\circ} 5$ y 17) se resolvió que los ejes serían dos enunciados afirmativos: la crisis frutícola con la desaparición de los productores pequeños y medianos, y la crisis lanera con el proceso de desertificación patagónica; actuarían como hilos conductores en el trabajo docente respetando cada disciplina su autonomía sin subordinaciones de ninguna índole.

Uno de los textos empleados en forma interdisciplinaria al comienzo del año lectivo con la apertura del ciclo escolar apuntaba a una definición muy escueta de Ciencias Sociales, en base al cual se presentaban las tres disciplinas del Área Mundo Socio-Cultural; y esto posibilitaba el trabajo de cada profesor en sus respectivas horas cátedras.

Las Ciencias Sociales son las distintas disciplinas que se encargan de estudiar la conducta de los hombres en su relación con los demás y con el medio, como así también su evolución en el tiempo y comportamiento ante distintos y determinados fenómenos. (Cuadernillo de Historia Regional, CBU N 15, 1989)

Asimismo, se analizaban textos cortos sobre el concepto de cultura; las primeras formas de organización del hombre y el concepto de región como un sistema abierto sin límites fijos, considerando al proceso productivo frutícola.

(...) El proceso productivo frutícola está integrado por distintas actividades vinculadas e interrelacionadas entre sí: chacra, galpones de empaque, frigoríficos, aserraderos, papeleras, jugueras, etc. Estas actividades se encuentran en distintas áreas del Alto Valle, es decir que se pueden localizar tanto en la provincia de Río Negro como en la provincia del Neuquén. Por ello, es importante señalar que una región no posee límites fijos; su alcance va más allá de los límites provinciales, dado que abarca además de Río Negro parte de la provincia del Neuquén. [subrayado propio].(Ferreira, 1991)

De esta manera, desde cada asignatura se introducía someramente en su objeto de estudio y metodología de trabajo. No debemos obviar el notable crecimiento y los debates teóricos de las Ciencias Sociales en la segunda mitad del siglo XX, ricos en aportes en el terreno de la investigación que reflejaron un acercamiento de las distintas ciencias y un desarrollo específico de cada una. 
Se debía vehiculizar nuevas visiones del pasado, actitudes y valores con respecto a las poblaciones originarias, el trabajo de los inmigrantes y el acceso a las tierras, las características de la élite, entre otros tópicos. Los equipos de docentes tomaron la iniciativa de dotar a estos textos de un contenido de tolerancia, eliminando posturas que pudieran fomentar las diferencias e incluso la discriminación hacia otras comunidades o etnias autóctonas. Se debía romper con la cristalización de una imagen del pasado nacional que se había convertido en el "sentido común" de nuestra historia. Algunas de las ideas e imágenes que circularon y estaban disponibles en este período se ponían de manifiesto en textos como el siguiente:

(...) En 1879 empezó en la Pampa y el norte de la Patagonia la mal llamada "Conquista o Campaña del Desierto" (mal llamada porque ésos no eran desiertos, sino tierras ocupadas por los indios). En un primer momento la cosa no afectó a los aonikenk de Chubut y Santa Cruz que vivían muy lejos. Pero en 1883, las expediciones militares llegaron hasta el sur de la Patagonia y algunas familias tehuelches fueron capturadas y enviadas por barcos a Buenos Aires. ¿Cómo era posible que se hiciera eso, cuando no existía aquí si siquiera el pretexto de estar en guerra con estos indios? Es que en 1879 un decreto del presidente Nicolás Avellaneda establecía el "reparto de indios": las familias de indios quedaban bajo custodia del Defensor Nacional de Pobres e Incapaces, quién decidía donde "colocar" a los indios para que trabajaran con familias que se comprometían a darles de comer, vestirlos, educarlos y pagarles un sueldo. Dejando de lado que los tehuelches tenían su propia forma de trabajar y que durante siglos se habían arreglado perfectamente para vestirse, comer y educar a sus hijos, en la práctica todo aquel palabrerío de la ley se traducía en que gente acomodada con el Gobierno recibía hombres, mujeres y chicos indios para trabajar como peones o sirvientes, a cambio de un plato de comida. Y no siempre se mantenían juntas las familias, que a veces se separaban para no verse más (...). (Palermo, 1993 a, p. 56).

Como se puede desprender de la lectura, los términos "indio", "indígena", "aborigen" se utilizaron indistintamente hasta su replanteo que se impuso al ir cambiando la mirada hacia el otro, en base a las redes personales, nuevas producciones, y el devenir del tiempo, e ir incorporando las categorías de "nativos", "comunidades originarias" o "pueblos autóctonos".

Las categorías de "desierto" y de espacio "vacío" resultaron cuestionadas por el Área "Mundo Socio-Cultural". Se intentó superar la imagen de la Patagonia de principios de siglo inserta en la clásica conceptualización: "civilizada/ incivilizada", dejar de analizar a las poblaciones originarias como "salvajes" en el contexto de la "conquista", como "bárbaros" en un territorio transformado. 
En el siguiente párrafo se puede inferir la importancia de los cuestionamientos a verdades inmutables y el incentivo a tomar posición frente a conflictos generados a partir del choque cultural y de intereses.

(...) Los productos de mayor comercio eran el ganado y la sal. Extinguido el ganado cimarrón, los indios comenzaron a arrear el manso que los hacendados habían aquerenciado en sus estancias. Difícil es saber de qué lado comenzaron las hostilidades. Podría pensarse que partió de los mapuches, necesitados de los animales con el fin de mantener su comercio con Chile, en donde recibían trigo, maíz y otros artículos. Pero también habría que culpar a los primitivos estancieros que continuamente invadían las tierras de los indios, ignorando los tratados y cometiendo con ellos toda clase de fechorías con lo que provocaban su lógica reacción. (Herrero y Salerno, 1989)

En 1992 siguiendo la línea de trabajo de Silvina Gvirtz (citado en Fernández, Lewkowicz, Mussi y Rodríguez, 2001) se comenzaba a abordar el problema del "otro", de la alteridad y los prejuicios con que es tratado, se intentaba presentar una visión distinta del nativo, gaucho, e inmigrante, actores sociales insertos en problemáticas concretas, algunas focalizadas en el tiempo y otras arrastradas hasta el presente. Por ello, desde Educación Cívica se analizaban textos periodísticos sobre la situación actual de las comunidades indígenas, los mapuches en Ruca Choroi, 12 de Octubre ¿festejo o tristeza?, entre otros. La intencionalidad educativa apuntó a la inclusión social del indígena, como sujeto histórico o actor social cuya incorporación social destierre la inferioridad, la marginalidad y pobreza, incluyéndolos como ciudadanos integrados al colectivo social. Tanto desde la Historia como de la Educación Cívica se trató de contextualizar el proceso de genocidio, destierro y explotación laboral de las distintas etnias, enfatizando en el proceso histórico periodizado para su análisis.

(...) la guerra duró pocos meses. Muchas tribus consiguieron escaparse hacia la Cordillera e incluso a Chile; muchos murieron en combate o fueron fusilados y los restantes fueron capturados. Algunas tribus fueron instaladas en "reservas" y otras separadas y su gente repartida: los hombres como peones de estancias, mujeres y chicos como sirvientes. Otros fueron llevados a Tucumán, a juntar cañas de azúcar, y también hubo quiénes terminaron en la isla Martín García, picando piedras para hacer adoquines para la ciudad de Buenos Aires. Así acabó la llamada "Conquista del Desierto", un nombre muy mal puesto porque en realidad no se conquistó ningún desierto (en los desiertos no vive nadie, ¿no?). (Palermo, 1993 b, p. 59)

(...) La forma que lo arriaban...uno si se cansaba por ahí, de a pie todo, se cansaban lo sacaban el sable lo cortaban en lo garrone. La gente que se cansaba y ... iba de a pie. Ahí quedaba nomá, vivo, 
desgarronado, cortado. (...) Yo me recuerdo bien por lo que contaba mi pobre viejo paz descanse (...), (Perea, 1989).

El tópico de las "campañas militares de 1879.1885" sirve para indagar la forma en que son presentados temas centrales de nuestra historia. En esta reconstrucción de las primeras experiencias se comenzaba con un texto titulado "Las rastrilladas Camino de los Chilenos" donde se presentaba a la zona de la Confluencia como un lugar de paso o vía de circulación de los mapuches; los textos siguientes se titulaban "El avance del hombre blanco", "La incorporación de los Territorios del Sur (1879-85)". Resultaba imprescindible el tratamiento de conceptos tales como "Revolución Industrial Inglesa", "División Internacional del Trabajo", "Oligarquía Terrateniente", "modelo agro-exportador argentino" porque constituían el marco o contexto histórico para insertar las campañas militares al mal Ilamado "desierto" entre 1789 a 1885 aproximadamente. De esta manera, la nueva Historia Económica y Social que se desarrollaba en el ámbito académico comenzaba a permear en estos textos que eran las herramientas de trabajo cotidianas en el aula (Lanza y Finocchio, 1993). Párrafos como los que se presentan a continuación se reiteraban a lo largo de varios textos explicativos:

(...) Estos territorios sustentaban una alta población aborigen considerada "bárbara" en contraposición a los hombres civilizados del siglo XIX. Estas tierras eran codiciadas no por el ganado cimarrón ya extinguido o por la sal sino para desarrollar el ganado bovino y la agricultura en la zona pampeana y criar ovejas Merino en los campos patagónicos. La invención del frigorífico en la década del 80 había permitido la exportación de carnes que junto a los cereales y la lana eran los principales rubros comprados por los británicos. En estas condiciones, la Argentina se incorporó al mercado mundial como país abastecedor de bienes primarios. (...). (Herrera y Salerno, 1989) (...) En la última década del siglo XIX, el Estado controlado por los terratenientes, cuyos intereses coincidían en general con los británicos, encaró la realización de las obras públicas como el sistema de riego y la creación de una infraestructura de transporte (ferrocarriles, puertos, etc...) orientado también por el esquema de la División Internacional del Trabajo que asignaba a la Argentina una especialización agro-exportadora (...). La expansión de la red ferroviaria constituyó la prueba de dinamismo de la economía exportadora. Fue construida en abanico alrededor del puerto de Buenos Aires que permitió integrar progresivamente todas las zonas productivas del país con el mercado internacional (...). (Rouquié, 1984)

Tales temáticas se trataban sin caer en el enciclopedismo, e impregnadas de un enfoque procesual para poder llegar al abordaje de los circuitos 
producticos de la alfalfa (1911-1930) y de la fruticultura (1930 en adelante), la desaparición paulatina de las explotaciones agropecuarias en un proceso de crisis tanto en el Alto Valle como en la región patagónica (Vapnarsky, 1983; Ockier, 1987).

Las explicaciones debían ser simples en la forma de expresarlas pero enfatizando en la complejidad de las causas históricas. Asimismo, estaba presente la importancia del contexto histórico en la escala regional y continental; considerar a la Argentina como parte de la realidad latinoamericana, del Tercer Mundo; esto formó parte de discusiones grupales en las instancias de perfeccionamiento docente, así como el nivel de complejidad que tendría el abordaje de tales temáticas para aquellos dispuestos a enseñarlos.

Además, se emplearon los juegos de simulación como estrategias para analizar problemáticas desde la mirada de distintos actores sociales (Zenobi y Ferrero, 1993), videos educativos, imágenes fotográficas, planos y mapas, artefactos domésticos de época, testimonios orales y escritos como el testimonios de pobladores autóctonos/originarios, artículos periodísticos y salidas de campo, en el medio local, con trabajos interdisciplinarios. Estas modalidades se encuadraban en la instancia de los Talleres Integrales en los cuales las áreas desarrollaban un proyecto de trabajo anual empleando el aula taller como estrategia de aprendizaje. Asimismo, se recurrió a la historia oral incentivando a los alumnos a realizar durante el receso invernal entrevistas a los abuelos o personas mayores oriundas o simplemente conocedores de la localidad. Estos informes que podían elaborarlos en forma individual o grupal se empleaban en sucesivas clases reconstruyendo la historia local, por décadas, con el aporte de todo el alumnado, compartiendo no sólo información sino fotografías de época.

\section{Reflexiones Finales}

El presente trabajo es una primera aproximación parcial e imperfecta, en consecuencia no se agota en esta instancia. El estudio se ha orientado al estudio de los textos escolares como herramientas de construcción y socialización discursiva, a partir de los cambios logrados en el marco de la Reforma del Nivel Medio en Río Negro. Se intentó centrar el análisis en las imágenes y las representaciones que sobre la región, la visión del "otro", los procesos de cambio a nivel de estructura socio-económica y temas superestructurales vinculados a la historia de las ideas, de las prácticas culturales se fueron forjando, en aquellas experiencias.

Para la época del C.B.U., no sólo hubo una ausencia de libros de textos sino el divorcio entre ellos y los avances de la historiografía académica, la nueva Geografía Social y los nuevos enfoques de convivencia y pluralismo. Y fue en el marco de la Reforma del Nivel Medio que se iniciaron los impulsos para superar las falencias del retraso y la desactualización de los contenidos y poder vehiculizar la comprensión de los procesos relacionados con proble- 
máticas del presente. Considero importante enfatizar que 1990 constituyó un punto de inflexión que dio comienzo a nuevas propuestas editoriales. El campo de la reflexión pasa por estos artefactos culturales que según Adriana Fernández Reiris (2005) permiten configurar la escolarización siendo una importante herramienta teórica para explorar el pasado y el presente de la escuela en relación con la sociedad y la cultura. Esta interpretación coincide con el enfoque de André Chervel (1995) quien enfatiza en la capacidad de toda institución para producir saberes específicos y lograr una cultura escolar que emerge del propio funcionamiento escolar y cuyos efectos se extienden en un proceso de configuración y reconfiguración.

\section{Referencias Bibliográficas}

- $\quad$ Achilli, E., Ageno R. y Ossana E. (1988) "Investigación de la práctica docente en Taller de Educadores" en Cuadernos de Formación Docente 4. Universidad Nacional de Rosario -UNR-

- Allal, L. (1979) Estrategias de evaluación formativa: concepciones psicopedagógicas y modalidades de aplicación Universidad de Ginebra. Perrenoud (eds.),Traducción de María José Montón

- Casávola, H. y Castorina J. A (Comp. 1980) "El rol constructivo de los errores en la adquisición de los conocimientos. Aportes para una teoría de los aprendizajes" en Aprendizaje hoy, año $1, \mathrm{~N}^{\circ} 2$.

- $\quad$ Clementi, H. (1991) La Patagonia, Libros del Quirquincho, Coquena SRL., (1). Buenos Aires.

- Chervel, A. (1995) "Historia de las disciplinas escolares: reflexiones sobre un campo de investigación”. Revista de Educación 295, 59-111.

- Bistuer, L., Nuñez P., Herrero A. y Salerno N. (1988-90) "Las Ciencias Sociales" en Cuadernillo de Historia Regional con textos seleccionados para $1^{\circ}$ año del CBU, Manuscrito no publicado.

- $\quad$ "Cuadernillo de Historia Regional con textos seleccionados para $1^{\circ}$ año del CBU" (1990). En Centro Provincial de Documentación e Información Educativa, Consejo Provincial de Educación de Río Negro, Viedma, y difundido a las escuelas mediante boletín "Para Agendar".

- Dobaño Fernández P., Lewkowicz M. , Mussi R. y Rodríguez M., (2001) "Los libros de texto como objeto de estudio: un balance de la producción académica 1983-2000" en Rodriguez M. y Dobaño Férnandez P. Los libros de texto como objeto de estudio. Ed. La Colmena. Buenos Aires.

- $\quad$ Fernández Reiris, A. (2005) La importancia de ser llamado "libro de texto". Hegemonía y control del currículum en el aula. UNPA. Miño y Dávila, Buenos Aires.

- $\quad$ Ferreyra, P. (1991) "EL hombre como modificador del paisaje" en Cuadernillo de Historia Regional CBU N¹7. Manuscrito sin publicar.

- Herrero, A. y Salerno N. (1989) "La rastrillada Camino de los chilenos o de la sal", "El mal llamado desierto" en Cuadernillo de Historia Regional, CBU N 15, 1989 con modificaciones posteriores. Manuscrito sin publicar.

- Lanza, H. y Finocchio S. (1993) Curriculum presente, Ciencia ausente. La enseñanza de la Historia en la Argentina de hoy. Tomo III Miño y Dávila Editores. Buenos Aires. 
- $\quad$ Linares, M. C. (2009) "Las editoriales a fines del siglo XX y su impacto en los libros de lectura escolar" en Bottarini R. y Spregelburd R. P. (Coord.), La alfabetización de un siglo a otro: desafíos y tendencias. UNLu y UNNe, Buenos Aires.

- Luc J. N. (1989) La enseñanza de la Historia a través del medio, Ed. Cincel-Kapelusz, (8), Bogotá.

- Moreira M. (1988) "Mapas conceptuales” en Rev Contactos, México.

- Novak J. (1980) La teoría del aprendizaje asimilativo de David Ausubel. Ed. Nueva Alianza, Madrid.

- $\quad$ Novak J. y Bob Gowin D. (1981) Aprendiendo a aprender, Ed. Martínez Roar, Madrid.

- Ockier, M. C. "Tierra, Relaciones Sociales y Caminos de Desarrollo en el Alto Valle de Río Negro". (Tesis) Universidad Nacional de Rosario, Mayo de 1987

- Oyola C. (Director, 1998) "Innovaciones educativas. Entre las políticas públicas y la práctica educativa. Un análisis de la Reforma Educativa del Nivel Medio en Río Negro (1986-1996)". Miño y Dávila, Buenos Aires.

- Palermo M. Á. (1993 a) "Los Tehuelches”, Colección "La otra historia” Vol. 8 (2) de Libros del Quirquincho. Coquena Grupo Editorial S.R.L., Buenos Aires.

(1990) "El verdadero nombre de los Onas, los Selknam" Colección "La otra historia" Vol. 3 (2) de Libros del Quirquincho, Coquena S.R.L., Buenos Aires.

(1991) “Los Yámanas”, Colección "La otra historia” Vol. 6 de Libros del Quirquincho, Coquena S.R.L., Buenos Aires.

(1993 b) "Los indios de la pampa", Colección "La otra historia" Vol 4 (3) de Libros del Quirquincho, Coquena S.R.L., Buenos Aires.

- $\quad$ Perea, Enrique J “.,...Y Félix Manquel dijo...”, Viedma, Biblioteca de la Fundación Ameghino, 1989. P. 6-7 y 18-19 en Palermo M. Á. (1993) "Los indios de la pampa", Colección "La otra historia" Vol 4, op cit, pág. 60 y 61.

- $\quad$ Rapoport M. (2003), Historia Económica, política y social de la Argentina (1880-2000), Ed. Macchi, (2), Buenos Aires. Pps. 858-1016.

- Revista "Puerta Abierta. Año 2. № 9" Abril 1986, Viedma (Río Negro). DC: Centro Provincial de Documentación e Información Educativa. Consejo Provincial de Educación de Río Negro.

- Rinaudo M. C. y Galvalisis C. (2002) Para leerte mejor... cómo evaluar la calidad de los libros escolares. La Colmena, Buenos Aires.

- $\quad$ Rouquié A. (1984) Poder Militar y Sociedad Política en la Argentina I. Hasta 1943. Emecé Editores, Buenos Aires.

- Rodríguez M. y Dobaño Fernandez P. (2001), Los libros de texto como objeto de estudio. Ed. La Colmena. Buenos Aires.

- Vapnarsky C. (1983) Pueblos del Norte de la Patagonia 1779-1957. Centro de Estudios Urbanos y Regionales (CEUR). Bs. As. Río Negro S.A, Río Negro.

- Zenobi V. y Ferrero A., (1993) Caminos, ferrocarriles y poblamiento Fundación Antorchas y CPE de Río Negro, FLACSO, Buenos Aires. 